\title{
Fatores Relacionados à Adesão ao Exame de Papanicolau entre as Mulheres de 18 a 59 anos
}

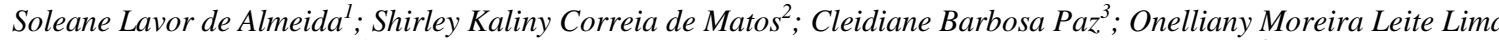 \\ Santana $^{4}$; Ana Cristina Mortimer Lio de Carvalho ${ }^{5}$; Jailma dos Santos Barbosa ${ }^{6}$
}

\begin{abstract}
Resumo: A pesquisa enfatiza sobre o que leva as mulheres a aderirem ao exame de Papanicolau, como também a periodicidade da realização do exame, o conhecimento que elas têm sobre o assunto e aqueles fatores que influenciam na não adesão ao exame em questão. O estudo tem como objetivo identificar os fatores que levam as mulheres a aderirem ao exame de Papanicolau. Trata-se de uma pesquisa de natureza descritiva, exploratória, com abordagem quantiqualitativa. O estudo teve como cenário a Unidade Básica de Saúde (UBS) do bairro Romeirão da zona urbana do município de Juazeiro do Norte, CE, onde abrangem a Equipe de Saúde da Família (ESF) 17. A pesquisa foi desenvolvida durante o ano de 2010. A população do estudo foi constituída por mulheres usuárias da UBS do município de Juazeiro do Norte, CE. A amostra foi composta pelas usuárias das ESF já citada, com faixa etária entre 18 e 59 anos de idade. O instrumento da coleta de dados foi uma entrevista semiestruturada, realizada nas UBS com aquelas que aderiram o exame de Papanicolau. Utilizou-se para analise dos dados qualitativos a técnica de caracterização de dados através das falas das participantes, após seu consentimento. Com relação às variáveis quantitativas, estas foram apresentadas na forma de tabelas e porcentagens. Evidenciou-se ao traçar o perfil socioeconômico que a maioria das entrevistadas encontrou-se na faixa etária entre 18 e 28 anos, predominando as casadas, de nível escolar baixo e as de renda mensal de um salário mínimo. Com relação ao perfil da periodicidade, prevaleceram principalmente aquelas que não recordavam a quantidade de exames de Papanicolau já realizados, aquelas com alguma alteração no resultado do exame e aquelas que dizem realizar o exame apenas se sinal / sintoma aparente. Na categoria conhecimento das mulheres acerca do exame de Papanicolau, prevaleceram aquelas que afirmaram que o exame é de diagnostico, poucas relataram que além de diagnostico é também de prevenção. Quando questionadas sobre os fatores que influencia a não adesão do exame, as mais verbalizadas foram, em ordem crescente: timidez, temor ao resultado e incomodo durante o procedimento. Já em relação aos fatores que levam a serem submetidas ao exame, prevaleceram aquelas que dizem procurar quando estão apresentando algum sinal / sintoma, poucas relataram que procurou a unidade sem qualquer sinal/sintoma. Indiscutivelmente nota-se a escassez de uma busca ativa ou mesmo da divulgação da importância do exame de Papanicolau e de seu objetivo principal que é o de prevenir e não diagnosticar, como os entrevistados tem o conhecimento. Portanto, vê-se a necessidade de órgãos responsáveis para uma melhor divulgação e por parte da atenção básica, na pratica educativa e busca ativa daqueles faltosos.
\end{abstract}

Palavras-Chave: Adesão; Exame Papanicolau; Estratégia Saúde da Família.

\section{Factors Related to Accession Papanicolau Test Among Women from 18 To 59 Years}

\begin{abstract}
The research focuses on what leads women to join the Pap smear, as well as the timing of the examination, the knowledge they have on the subject and those factors that influence non-adherence to the examination in question. The study aims to identify the factors that lead women to join the Pap smear. This is a descriptive research and exploratory, with quant. The study was the scene of UBS Romeirão neighborhood of the urban area of Juazeiro do Norte - CE, which covers the FHT 17. The research was conducted during the year of 2010. The study population consisted of female clients of UBS's northern city of Juazeiro - CE. The sample was composed by the users of the ESF quoted, aged between 18 and 59 years of age. The instrument of data collection was a semi-structured interview, held with UBS in those who joined the Pap smear. It was used for

\footnotetext{
1

${ }^{2}$ Graduação em Enfermagem. Especialização em Saúde Coletiva com Enfase em PSF, pela Faculdade Santa Emília de Rodat, FASER, Brasil ${ }^{3}$ Graduação em Enfermagem. Especialização em Enfermagem em Unidade de Terapia Intensiva pela Universidade Estadual Vale do Acaraú, Brasil. 4

${ }^{5}$ Psicóloga. Coordenadora de Recursos Humanos na Ae C Contact Center.

${ }^{6}$ Graduada em Enfermagem e Pedagogia. Especialista em Gestão em Saúde, Especialista em Auditoria em Saúde, Especialista em Enfermagem do Trabalho, Especialista em Saúde da Família, Especialista em Gestão Escolar. E-
} 
qualitative data analysis technique to characterize the data through the words of participants, after informed consent. Regarding the quantitative variables, these were presented in tables and percentages. It was clear to draw the socioeconomic profile that most subjects found in the age group between 18 and 28 years, mostly married women of low educational level and monthly income of a minimum wage. Regarding the profile periodicity prevailed especially those who did not recall the number of Pap tests already carried out, those with a change in the result of the examination and those who say take the examination only if signs / symptoms apparent. In the category of women's knowledge about Pap smear, those who prevailed said the test is diagnostic, a few reported that in addition to diagnosis is also prevention. When asked about the factors that influence nonadherence of the examination, most expressed were, in ascending order: shyness, fear of the result and discomfort during the procedure. In relation to the factors leading to their consideration, those that prevailed when they say look showing some signs / symptoms, few reported that they sought unity without any sign / symptom. Arguably one notice the lack of an active search or dissemination of the importance of Pap smear and their main goal is to prevent and not diagnose, as the respondents have the knowledge. Therefore, we see the need for responsible agencies to better disclosure and by the primary care practice in education and active search of those missing.

Keywords: Adhesion; Pap smears; Family Health Strategy

\section{Introdução}

Atualmente no mundo, as ações voltadas à saúde estão centradas na teoria: melhor que tratar é buscar uma forma para prevenir o desenvolvimento de doenças, e em alguns casos quando a prevenção não é tão eficaz ou o único meio para isto, tenta-se a detecção precoce de lesões já conhecidas como responsáveis mais adiante por aumentar as probabilidades da manifestação de patologias graves.

Uma doença de grande escala é o câncer de colo do útero, muito discutido principalmente por poder ser detectado precocemente com a realização periódica anual do exame de Papanicolau. Apesar da divulgação do assunto nas unidades de saúde e nos vários meios de comunicação, ainda pode-se encontrar mitos e medos em algumas mulheres para realizar o exame preventivo - é fato o argumento de que muitas delas desconhecem sua importância e por isso não procuram as unidades básicas de saúde para fazê-lo.

Em 1984, foi instituído o Programa de Atenção Integral à Saúde da Mulher (PAISM), que representou um marco histórico das políticas publicas dirigidas as mulheres. O Programa Viva Mulher que teve o projeto-piloto implantado em 1997, foi criado pelo ministério da saúde com o intuito de reduzir a mortalidade e as consequências psicossociais que o câncer de colo de útero pode causar às mulheres brasileiras (YASSOYAMA; SALOMÃO; VICENTINI, 2005). O Câncer de Colo Uterino (CCU) é a segunda causa de morte por câncer entre mulheres no mundo, com maior incidência nos países em desenvolvimento. No Brasil representa o terceiro mais comum tipo de neoplasia maligna que acomete mulheres depois do câncer de pele não melanoma e do câncer de mama (FERNANDES et al., 2009). A Organização Mundial de Saúde (OMS) assinala os fatores sociais, ambientais e hábitos de vida como os de maior incidência para a patologia, destacando condições socioeconômicas 
precárias, início precoce da atividade sexual, multiplicidade de parceiros, tabagismo, precárias condições de higiene e uso prolongado de contraceptivos orais. (DAVIM et al., 2005).

As Doenças Sexualmente Transmissíveis (DST's) configuram outro fator de risco de grande significância, principalmente na exposição ao Vírus Papiloma Humano (HPV), cujo estudo vem demonstrando seu papel importante no desenvolvimento das neoplasias de células cervicais e na sua transformação em células cancerígenas. Estando o HPV presente em 99\% dos casos de câncer de colo de útero, a idade é tida como fator de risco, sendo a faixa etária de maior incidência a de 35-49 anos de idade, com destaque para aquelas mulheres que nunca realizaram o exame de Papanicolau (DAVIM et al., 2005).

Com a realização do exame preventivo as alterações no colo do útero podem ser identificadas e tratadas, antes da manifestação da doença. Ações que visem reduzir os fatores de risco, principalmente tabagismo e infecção pelo HPV, devem ser estimuladas.

Devem-se submeter ao exame preventivo, as mulheres com vida sexual, principalmente aquelas entre 25 e 59 anos de idade. Mulheres grávidas também podem fazer o exame. Os cuidados antes da realização são: não estar menstruada, nos dois dias anteriores ao exame, não ter relação sexual, mesmo com camisinha e não usar duchas ou medicamentos vaginais (INCA, 2009). Nisto, com base nessa contextualização, questiona-se: Quais os motivos que levam as mulheres aderirem ao exame e os fatores que dificulta essa adesão?

Através de estágios em unidades de saúde da Estratégia de Saúde da Família (ESF) observamos que a grande maioria das mulheres só procura realizar o exame de Papanicolau, quando suspeita de uma possível Doença Sexualmente Transmissível (DST), muitas delas não tem conhecimento sobre o assunto e desconhecem a importância da periodicidade deste.

Diante disto, veio então o desejo de realizar um estudo que contribua na construção do perfil das mulheres que buscam com maior frequência a realização do exame preventivo do câncer de colo de útero, quais fatores estão relacionados à adesão das mulheres, ao exame preventivo, podendo com isso dar uma atenção maior aquelas que não aderem ao exame, estando-as mais susceptíveis à doença.

O objetivo deste estudo é identificar os fatores que levam as mulheres a aderirem ao exame de Papanicolau. Onde por meio deste, esperou-se também: 1) Avaliar o perfil socioeconômico das mulheres que aderem ao exame de Papanicolau; 2) Mensurar a periodicidade da realização do exame de Papanicolau; 3) Avaliar o conhecimento das mulheres em relação ao exame de Papanicolau; e 4) Identificar quais fatores propicia a adesão ao exame Papanicolau pelas mulheres. 


\section{Referencial Teórico}

O exame de prevenção do Câncer de Colo Uterino foi formulado na década de 1930, pelo Dr. George Papanicolau. Tal exame é realizado em nível ambulatorial e não provoca dor. No entanto, pela própria natureza do exame, que envolve a exposição de órgãos relacionados à sexualidade, o Papanicolau, é motivo de desconforto emocional para muitas mulheres (GREENWOOD; MACHADO; SAMPAIO, 2006).

Embora o exame citopatológico tenha sido introduzido no Brasil, na década de 1950, a primeira mobilização nacional para detecção precoce da doença ocorreu em 1998, onde foram colhidos três milhões de exames, dos quais $65 \%$ foram na faixa etária de 35 a 49 anos de idade, onde foram detectados 60 mil exames com alguma alteração, sendo acompanhadas e tratadas $77 \%$ das mulheres com lesões de alto grau e câncer (BRASIL, 2006), sendo que há mais de 30 anos, programas para detecção do câncer do colo uterino com base no exame Papanicolau (DUTILH; BRAGA; SCHOUT, 2006).

Em todos os países onde esse método foi implantado, houve uma redução na incidência e no índice de mortalidade pelo câncer do colo uterino, pois são identificadas as chamadas lesões "précancerosas" e as pacientes são adequadamente tratadas, eliminando-se o estigma da doença (IMEBI, 2010). Embora o Brasil tenha sido um dos primeiros países a implantar o exame de Papanicolau na rede de saúde pública, ainda assim, a segunda maior causa de óbito feminino por câncer continua a ser o do colo uterino. (SANTOS et al, 2007). Mesmo com o Programa de Assistência Integral à Saúde da Mulher (PAISM), criado em 1983, objetivando maior cobertura das ações voltadas à educação, ao diagnóstico precoce na tentativa de prestar uma assistência mais ampla e completa a mulher. (SOARES et al, 2010).

Avaliando a manifestação da patologia em questão, esta se apresenta inicialmente com uma fase pré-clínica assintomática, em que a detecção de possíveis lesões precursoras se dá através da realização periódica do exame preventivo anteriormente citado. Com o desenvolver da doença, os principais sintomas do câncer do colo do útero são sangramentos vaginais, corrimento e dor (INCA, 2009). É importante alertar para possíveis hemorragias ou sangramentos pós-coito, pequenos sangramentos sem causa aparente e leucorréia purulenta, sintomas que devem ser investigados. Já a dor pélvica, a perda de urina ou fezes pela vagina, perda de peso e anorexia são sintomas de câncer avançado (MORAES, 2007).

Pela pouca ou até mesmo nenhuma manifestação de sintomas no início do desenvolvimento de um câncer de colo uterino, é que boa parte das mulheres não procuram tratamento nesta fase. Ao avançar da doença a mulher começa a se queixar de ciclos menstruais anormais, secreção vaginal amarelada ou sanguinolenta, após ato sexual apresentar sangramentos e dores na parte inferior do abdômen, mais adiante estas dores se intensificam, pode ocorrer anemia pela perda de sangue, 
alterações da rotina intestinal, lombalgia, uremia importante, também hematúria e a dor pode se irradiar pela coluna lombar, membros inferiores e pela pelve. (RIVOIRE et al, 2006).

A relação entre câncer do colo uterino e os hábitos sexuais (promiscuidade, grande número de filhos, início precoce da atividade sexual e infecções ginecológicas repetidas) levou à identificação do Papiloma vírus Humano (HPV) como fator causal (LIMA; PALMEIRA; CIPOLOTTI, 2006). Esse tipo de câncer é na maioria dos casos associados a fatores extrínsecos, isto é, relacionados ao ambiente e aos hábitos de vida (tabagismo, ingestão deficiente de vitaminas, múltiplos parceiros sexuais, histórico de DST's, menarca precoce e uso de anticoncepcionais orais), infere-se assim a possibilidade de se empregar meios que afaste esses fatores de risco e em consequência diminua a incidência do câncer cérvico-uterino. Entretanto, não é fácil mudar os aspectos do estilo de vida de uma população, principalmente em meio à pobreza e a educação deficiente (LIMA; PALMEIRA; CIPOLOTTI, 2006).

Porém, afirma-se que a transformação neoplásica do epitélio cervical está relacionada com a idade do início da atividade sexual, com o número de parceiros sexuais, tabagismo, carências nutricionais, o número de gestações e de filhos, imunossupressão, infecção pelo vírus HIV, e sobre tudo, infecção pelo HPV (PIATO et al, 2005). Nisto, os fatores de risco estão relacionado às mulheres de condições socioeconômicas baixas, deficientes nível de escolaridade e de difícil acesso aos meios de saúde, como também a falta de conhecimento do assunto, medo e tabus de algumas mulheres.

Diante disto, o essencial que pode ser feito pelos profissionais de saúde, em seus diversos meios de atuação é a promoção à prevenção e disposição de um tratamento e acompanhamento da portadora de tal neoplasia. Quanto à prevenção primária pode ser uso de preservativos durante a relação sexual, sendo uma das formas de evitar o contágio pelo Papiloma vírus Humano (HPV), o qual tem importante papel no desenvolvimento no Câncer do Colo Uterino (CCU) e suas lesões precursoras (SOARES et al., 2010). Já quanto ao controle obedece à estratégia de prevenção secundária baseada na citologia cervical. Esta técnica de detecção, conhecida popularmente como Prevenção. O exame de prevenção do câncer cérvico uterino, além de sua importância para a saúde da mulher, é um procedimento importante de detecção precoce, de lesões invasivas e consequentemente, instrumento essencial para a diminuição da mortalidade por esta patologia (FERREIRA, 2009).

Em si, para ter uma ideia da importância e da valorização que o exame de prevenção possui no âmbito brasileiro, no ano de 1988, o país adotou a norma da Organização Mundial de Saúde, que propõe o controle do CCU nas mulheres com idade entre 25 e 60 anos, a cada três anos, após dois resultados negativos ou intervalo anual, contudo, estimativas indicam que cerca de $40 \%$ das mulheres brasileiras nunca fizeram o exame. Dentre as razões para a baixa adesão está: a natureza do exame, que envolve exposição da genitália, razão de desconforto emocional para algumas clientes, em virtude de pudores e tabus, além das condições socioeconômicas baixas e falta de conhecimento sobre a doença (FERNANDES et al., 2009). 
O tratamento das pacientes portadoras do câncer do colo uterino consiste na cirurgia, radioterapia e quimioterapia. O tratamento a ser realizado depende das condições clínicas da paciente, do tipo de tumor e de sua extensão. No estágio inicial do tumor, os resultados da cirurgia radical e da radioterapia são equivalentes.

O tratamento cirúrgico consiste na histerectomia (retirada do útero), porção superior da vagina e linfonodos pélvicos. Os ovários podem ser preservados nas pacientes jovens, dependendo do estadiamento do tumor; quanto mais avançado, mais extensa é a cirurgia.

O tratamento radioterápico pode ser realizado como tratamento exclusivo, ou ainda pode ser feito associado à cirurgia (precedendo-a), ou quando a cirurgia é contraindicada (MORAES, 2007).

Por fim, se há uma intensificação das ações de conscientização da população feminina para a realização do exame Papanicolau, há também a possibilidade de diminuir os índices de óbitos entre as mulheres, por conta do câncer de colo de útero. Todavia, quando se diz "intensificação das ações de conscientização", afirma-se que estas não devem estar centradas apenas em convidar a cliente a fazer o exame, mas apresentar as razões do mesmo, e mais ainda, explicar a importância de fazê-lo, independente do gênero do profissional que fará a coleta (devido o receio que algumas mulheres possuem), da condição socioeconômica e do nível escolar da cliente, ou ainda da idade que esta possui se já estiver em idade fértil ou no inicio de suas atividades reprodutivas. Estes são os desafios que devem ser superados para uma melhor apresentação dos índices de casos registrados e tratados, com sucesso, em nosso país.

\section{Método}

O presente estudo teve como proposta metodológica a pesquisa de natureza descritiva, exploratória com abordagem quantiqualitativa, assegurando assim a obtenção dos objetivos propostos. A pesquisa em questão se deu na Unidade Básica de Saúde (UBS), da rede pública, mais especificamente na Estratégia de Saúde da Família (ESF) nº17, situada no bairro Romeirão, na zona urbana do município de Juazeiro do Norte, cidade localizada no interior do Estado do Ceará, atualmente com 64 Equipes da Estratégia Saúde da Família, divididos em cinco distritos sanitários, atendendo a uma população superior a 250.000 mil habitantes. A pesquisa foi desenvolvida durante o ano de 2010 , onde a coleta de dados foi realizada durante os meses de setembro e outubro, seguindo as etapas de desenvolvimento, análise e finalização.

A população do estudo foi constituída por mulheres usuárias da Unidade Básicas de Saúde do Município de Juazeiro do Norte, CE. A amostra foi composta pelas usuárias da Estratégia de Saúde da Família, $n^{\circ}$ 17, do bairro Romeirão, na faixa etária entre 18 a 59 anos. O número total desta foi de 12. Os critérios de inclusão foram: adesão do exame de Papanicolau; residir na zona urbana; ser usuária da 
ESF 17; ter entre 18 e 59 anos; e estar em plena aptidão física e/ou mental. Os critérios de exclusão foram: não adesão ao exame Papanicolau, residir na zona rural; realizar o exame em outra unidade de saúde; não encontrar-se entre a faixa etária de 18 a 59 anos; e apresentar alguma alteração em sua aptidão física e/ou mental.

O instrumento da coleta de dados foi uma entrevista semiestruturada realizada na Unidade Básica de Saúde (UBS) com as mulheres que aderiram ao exame Papanicolau. Para a análise dos dados foi utilizado o método de análise do conteúdo que nada mais é do que uma forma de avaliar o que foi relatado pelos pesquisados de uma forma sistemática e organizada, para que se alcancem os objetivos traçados na pesquisa. (BARDIN apud. LEOPARDI, 2002). Já, para a análise dos dados qualitativos, foi utilizado à técnica de categorização dos dados, através das falas das participantes. Logo, com relação às variáveis quantitativas foi apresentada na forma de tabelas, no programa Microsoft Office Windows versão 2007, para melhor exposição dos dados e de fácil entendimento para o leitor.

Legalmente, a pesquisa se deu conforme dispõe a Resolução nº 196, de 10 de outubro de 1996, do Conselho Nacional de Saúde, a respeito dos preceitos éticos da pesquisa, onde a participação dos sujeitos se dava após a aceitação dos mesmos com a assinatura em um Termo de Consentimento Livre e Esclarecido, o qual trazia a garantia do sigilo e anonimato das informações e dados coletados, bem como a desistência ou não da pesquisa. Sendo assim, a pesquisa respeitará os aspectos éticos da pesquisa científica, que são: não maleficência, respeito à pessoa e a justiça, principalmente em relação ao artigo $3^{\circ}$, que se refere aos aspectos éticos da pesquisa envolvendo seres humanos.

\section{Análise dos Dados}

O primeiro ponto apresentado neste estudo, quanto a resultados na coleta de dados, é o perfil socioeconômico das mulheres que aderem ao exame Papanicolau, e que estavam sendo assistidas pela Equipe da $17^{\circ}$ Unidade de Saúde da Estratégia Saúde da Família. Este traço se encontra registrado na Tabela 1.

Tabela 1: Perfil socioeconômico das mulheres que aderem ao exame de Papanicolau, assistidas na ESF do bairro Romeirão. Juazeiro do Norte, CE, 2010.

\begin{tabular}{l|l|c}
\hline \multicolumn{1}{c|}{ VARIÁVEIS } & $\mathbf{N}^{\mathbf{0}}(\boldsymbol{\%})$ \\
\hline \multirow{5}{*}{ Faixa Etária } & $18-28$ & $05(41,66 \%)$ \\
& $29-39$ & $03(24,99 \%)$ \\
& $40-49$ & $03(24,99 \%)$ \\
& $50-59$ & $01(08,33 \%)$ \\
\hline \multirow{3}{*}{ Estado Civil } & Casada & $11(91,63 \%)$ \\
& Solteira & $01(08,33 \%)$ \\
& Divorciada & $00(00,00 \%)$ \\
\hline
\end{tabular}

70 Id en line Revista de Psicologia. Ano 9, No. 27. Julho/2015 - ISSN 1981-1179. Edição eletrônica em http://idonline.emnuvens.com.br/id 


\begin{tabular}{c|l|c}
\hline \multirow{3}{*}{ Escolaridade } & Ensino Fundamental Incompleto & $08(66,64 \%)$ \\
& Ensino Médio Completo & $03(16,66 \%)$ \\
& Ensino Superior Incompleto & $01(08,33 \%)$ \\
\hline \multirow{3}{*}{ Profissão } & Dona de Casa & $05(41,66 \%)$ \\
& Autônoma & $02(16,66 \%)$ \\
& Outros & $05(41,66 \%)$ \\
\hline \multirow{2}{*}{ Renda } & < que 01 salário & $02(16,66 \%)$ \\
Familiar & 01 salário & $07(58,31 \%)$ \\
& $>$ que 01 salário & $03(24,99 \%)$ \\
\hline
\end{tabular}

Fonte: Pesquisa direta, 2010.

Diante da tabela 1 a faixa etária predominante entre as participantes foi de 18 a 28 anos de idade (41,66\%), o que evidenciou um fator positivo ao constatar maior preocupação entre as que fazem, pois segundo Costa (2007) o câncer ou carcinoma da cérvice e o câncer do colo uterino é o mais comum entre as mulheres com faixa etária de 35 a 50 anos. Cerca de $58 \%$ das participantes do estudo ( 3 entre 29 e 39 anos; 3 entre 40 e 49 anos e 1 com 59 anos) apresentam-se na faixa etária de maior incidência para o câncer de colo uterino, ou seja, idade de alerta (DAVIM et al, 2005).

Verificou-se que a maioria das participantes, $91,63 \%$, possui vínculo matrimonial, e apenas 01 $(08,33 \%)$ afirma ser solteira. O hábito sexual é um fator importante para a etiologia de cânceres e tumores ginecológicos. O papilomavírus é um importante agente etiológico nos CA de vulva, vagina e, principalmente, do colo uterino. A multiplicidade de parceiros sexuais contribui para o maior risco de contrair o vírus (BRASIL, 2005; COSTA, 2007). Entretanto, ressalta-se que existe uma tendência de solteiros sem parceiro fixo constituírem um fator de risco para o CA de colo uterino devido o papilomavírus (DAVIM, 2005), o que não prevaleceu na presente pesquisa.

Quanto ao grau de escolaridade, $66,64 \%$, ou seja, a maioria das mulheres da pesquisa não tem sequer o ensino fundamental completo. Apenas duas concluíram o ensino médio. A falta de escolaridade e de conhecimentos básicos se torna um obstáculo para essas mulheres sobre a importância do exame de prevenção do CA de colo uterino (FERREIRA, 2010). Por isso a necessidade e a importância da educação em saúde realizada nas unidades básicas de saúde pelos profissionais de saúde, em especial, o enfermeiro.

Quanto à profissão, prevaleceram aquelas que afirmaram ser dona de casa, 41,66\%. Apenas duas se disseram ser autônomas e as demais tem alguma profissão fora de casa, dentre elas: cabeleireira, comerciante e técnica de enfermagem, o que pode ser um obstáculo para não realização do exame preventivo em questão. No caso, em sua pesquisa, Ferreira (2009) observou que fatores que estavam associados ao papel da mulher (cuidado com a casa, filhos, dentre outros) influenciam na não realização do exame preventivo do câncer do colo uterino.

A classe econômica predominante foi as que vivem com cerca de um único salário mínimo, $58,31 \%$, apenas 03 das 12 entrevistadas tem renda maior que 01 salário mínimo e 02 sobrevivem com menos de um salário mínimo. Observam-se condições econômicas pouco favoráveis na maioria dos 
casos. Existe uma relação íntima entre o grau de escolaridade e classe econômica não favorável, fazendo com que mulheres enquadradas nesta relação sejam mais suscetíveis ao acometimento do câncer de colo uterino (DAVIM, 2005). Uma pesquisa feita por Duavy et al (2007) revelou que há uma associação entre o câncer cérvico-uterino e o baixo nível socioeconômico. Os grupos vulneráveis concentram-se onde existem as maiores barreiras de acesso à rede de serviços, diz o autor.

Em seguida, é apresentado o perfil da periodicidade ao exame das participantes na pesquisa. Estes dados estão dispostos na Tabela 2.

Tabela 2: Perfil da periodicidade ao exame das participantes usuárias da ESF do bairro Romeirão, Juazeiro do Norte, CE, 2010.

\begin{tabular}{c|l|c}
\hline \multicolumn{2}{c|}{ VARIÁVEIS } & $\mathbf{N}^{\mathbf{0}}(\boldsymbol{\%})$ \\
\hline \multirow{2}{*}{$\mathbf{N}^{\mathbf{o}}$ de Exames Papanicolau } & Não Lembram & $05(41,66 \%)$ \\
& $1^{\text {a } \text { Vez }}$ & $01(08,33 \%)$ \\
& Mais de 2 Exames & $06(49,88 \%)$ \\
\hline Alterações no Resultado do & Alguma Alteração & $10(83,03 \%)$ \\
Último Exame Papanicolau & Ausência de Alterações & $02(16,66 \%)$ \\
\hline Periodicidade em Realizar o & 6 em 6 Meses & $02(16,66 \%)$ \\
Exame & A Cada Ano & $04(33,32 \%)$ \\
& Quando Apresenta Algum Sintoma/Sinal & $06(49,88 \%)$ \\
\hline
\end{tabular}

Fonte: Pesquisa direta, 2010.

$\mathrm{Na}$ pesquisa houve um expressivo número de participantes que afirmou não se lembrar da quantidade de exames Papanicolau já realizados até a data da pesquisa, segundo a maioria, por ser uma prática difícil de ocorrer, $41,66 \%$ relataram não lembrar; $8,33 \%$ diz ser a primeira vez que se submeteu a esse tipo de exame e cerca de $49 \%$ realizaram um total de 2 a 5 exames preventivos de câncer do colo uterino. Nota-se a pouca procura por parte da maioria das participantes para a prevenção, mas não para o diagnóstico de doenças.

Evidenciou-se uma grande prevalência daquelas mulheres que já tiveram resultados do exame alterados, $83,3 \%$. As alterações mais comuns foram corrimento vaginal e inflamação, segundo as pesquisadas. Apenas duas relataram não ter qualquer alteração no resultado de seu último exame citopatológico. Ressalta-se que houve ausência de diagnósticos malignos.

A periodicidade dos exames, quando indagados, foi de 6 em 6 meses (16,66\%), a cada ano $(33,32 \%)$ ou quando alguma sintomatologia $(49,98 \%)$. Visualizando a não adesão ao exame para prevenção e sim como exame diagnóstico pelas entrevistadas.

Fazendo um paralelo entre o número de exames realizados, os resultados obtidos e a periodicidade em realizá-los, perceberam claramente que há uma divergência quando comparado às orientações preconizadas pelo Ministério da Saúde (MS), que diz: O exame de Papanicolau deve ser aderido por aquelas que se encontre entre a faixa etária de 25 anos e 59 anos de idade, ou antes, se já iniciou sua vida sexual. $\mathrm{O}$ exame preventivo tem periodicidade anual, inicialmente. Após 2 exames 
consecutivos com resultados negativos para displasia ou neoplasia do colo de útero, a mulher será submetida ao exame a cada 3 anos. Vale ressaltar que mesmo no resultado laboratorial sem alterações, mas a paciente refere algum sintoma e/ou é visualizado alguma ferida, por exemplo, a mulher deve ser tratada seguindo o protocolo do MS, ou seja, a clínica é soberana (BRASIL, 2005). O que se percebe é que para a maior parte das participantes da pesquisa, esse exame tem objetivo diagnóstico e não preventivo.

\section{Discussão}

O exame citopatológico ou teste de Papanicolau é um método simples, barato e eficiente para detecção de lesões desde sua fase inicial, e está à disposição da população feminina em toda rede básica de saúde (ALMEIDA et al, 2008). Segundo os autores, o assunto ainda não é tão divulgado quando comparado ao exame preventivo do CA de mama. A divulgação na mídia, palestras nas UBS e cartazes são meios de chamar a atenção das mulheres sobre a importância do exame de prevenção do CA colo uterino afirma Duavy et al (2007). Tendo em vista a realidade das mais diversas unidades de saúde, os autores afirmam ainda que a falta de orientação e/ou conhecimento sobre o exame é, sem duvida, um fator que interfere na não adesão das mulheres ao exame.

A partir dessa problemática, vê-se a necessidade de uma maior divulgação sobre o assunto em questão, ações educativas e divulgação na mídia seria um bom começo. Logo, se questiona de qual o conhecimento das mulheres acerca do exame Papanicolau e quem a informou sobre o assunto. Quando questionadas a respeito do conhecimento acerca do exame algumas integrantes relataram:

\footnotetext{
"Não sei nada... é a primeira vez que faço esse exame... Minhas irmãs me informaram, pois minha bisavó morreu de câncer no útero." ARIEL.

"Sei que serve para prevenir doença e corrimento... soube na escola, uma palestra..." MEBAHEL.

"Para descobrir alguma doença em tempo de cura... desde que tive o meu primeiro filho minhas amigas diziam que é importante fazer." MICAEL.

"Prevenir doença e ver se tem feridas... a agente de saúde que avisou que a enfermeira do posto tava chamando pra fazer o exame..." DANIEL.
}

As revelações acima demonstram a falta de conhecimento sobre, de fato, o que é e qual a finalidade. 10 das 12 entrevistadas relataram que o exame é para diagnosticar doença (s), ao contrário do seu objetivo, de acordo com o MS que é de prevenção (BRASIL, 2005). No caso, a pesquisa comunga-se com o estudo de Davim et al (2005) que afirma em seus resultados quando se refere à finalidade do exame Papanicolau, que na opinião das entrevistadas, 58\% diz que o exame previne 
contra doenças e principalmente o CA de colo uterino. Entretanto, pode-se considerar que apesar de as entrevistadas não terem um conhecimento aprofundado, pode-se afirmar que mesmo assim é satisfatório o pouco de conhecimento que tem.

Já em relação ao modo como adquiriram informações sobre o assunto em questão, prevaleceu aquelas que adquiriram algum tipo de conhecimento através de amigos e familiares; também se ressalta que algumas adquiriram este a partir de conversas com o ACS e enfermeiro (a) da unidade, apenas 2 das 12 participantes dizem não saber sobre o assunto abordado, onde temos como exemplo deste fato, a fala de Ariel.

Desde 1998 as Secretarias Estaduais e Municipais de Saúde vem sendo convidadas pelo Instituto Nacional de Câncer (INCA) e o Ministério da Saúde a promover ações regionais na busca da efetivação de um programa de rastreamento do CA de colo uterino, como também a educação em saúde, iniciada na atenção primária. Para que o programa atinja seu objetivo é fundamental uma rigorosa sintonia entre os profissionais envolvidos no mesmo (ALMEIDA et al, 2008). Pelas verbalizações de Micael e Ariel notou-se a ausência de atenção sobre o assunto por parte dos profissionais da ESF e que só Daniel referiu ter sido "chamada" pela enfermeira para realizar o exame. A ausência total do profissional médico do posto quanto ao assunto foi notória no estudo.

Em relação aos fatores que impedem a realização do exame Papanicolau, predominou aquelas que verbalizam ter vergonha, as que temem o resultado e/ou que sentiu/sentem incomodo na realização do mesmo. Respectivamente: 66,64\%, 66,64\% e 51,96\%. As mesmas entrevistadas que afirmam ter "vergonha" de ser submetida ao exame ou relataram tal sentimento, também afirmaram temer o resultado e/ou sentir incomodo (dor) o Papanicolau. Essas afirmações são apresentadas das seguintes formas:

\footnotetext{
"Muita... e é porque faço esse exame desde o meu primeiro filho e ainda não me acostumei... tenho medo do resultado e fico ansiosa para receber o resultado... sinto dor durante e ardência depois do exame... "VEHUEL.

"Não tenho vergonha e nem dói, mas tenho medo do resultado do exame". BENAEL.
}

"Sim! Mesmo sendo com a enfermeira mulher, só faço se for com uma mulher... tenho (medo do resultado). Sei que não dói, mas parece que toda vida vai doer." ANIEL.

"Tive na primeira vez que fiz... tenho sim (medo do resultado). Sempre incomoda, nem que seja um pouquinho, dói...” MENADEL.

"Não tenho vergonha mais não, mas sinto medo do resultado e se vai doer ou não...”. DANIEL.

Dentre os relatos, observou-se ainda que apenas 4 das 12 entrevistadas relataram não ter vergonha do exame, mas que temem o resultado. Outro fator impeditivo é o incomodo, relatado 
principalmente por Vehuel e Menadel. Mas o fator mais expressivo foi sem dúvida a timidez em realizarem o exame. O temor da desonra, vexame, o opróbrio, são nomenclaturas para o termo usado “vergonha/timidez" (TERSARIOL, 2000).

Em momento algum durante a entrevista foi relatada pelas entrevistadas a ausência de qualquer fator, percebeu-se que sempre prevalecia algo que a impedia de realizar o exame. Estudos como o de Oliveira et al (2006) e Ferreira (2009) confirmam também a prevalência desses fatores. Ferreira (2009) comunga-se com o presente estudo quando afirma que o fator "ter vergonha" em realizar o exame foi à justificativa mais referida para não realização do mesmo. Revelando assim o quanto a intimidade e a sexualidade influenciam, nesse caso negativamente, sobre a vida das mulheres, afinal, trata-se de ser tocada por um estranho em suas partes íntimas. Nisto, é importante o diálogo do profissional que realizará o exame citológico, independentemente se é ou não a primeira vez, para que a cliente reconheça a importância da realização deste e sintam-se mais a vontade, pois como relataram mesmo tendo feito várias este, ainda não se sentem a vontade ao realizarem o exame em questão.

Quanto aos demais fatores, temor ao resultado do exame e incomodo do mesmo, são assuntos pouco discutidos tanto por parte das pacientes como dos profissionais de saúde. Não foi verbalizado por qualquer uma das participantes a falta de recursos humanos e/ou materiais como um fator impeditivo para a realização do exame, o que não é verdade no estudo feito por Oliveira et al (2006) que evidenciou que um dos maiores fatores associados a não realização do exame preventivo de Papanicolau em São Luiz, MA, foi a falta de materiais e a dificuldade de acesso a unidade de saúde.

A dor ao exame citológico é relativa, depende de como se encontra a mulher naquele momento: tensa, nervosa ou relaxada. Quando a paciente encontra-se relaxada o espéculo é introduzido com maior facilidade, assim o tempo para realizar o exame é reduzido consideravelmente, diminuindo o provável incomodo relatado por elas (DUAVY, 2007).

Se a mulher chegar à UBS com afirmações negativas sobre o exame ou mesmo dúvidas e não são informadas ou esclarecidas, fica claro que não é possível mudar a repercussão "maléfica" do exame para essas mulheres. Nas falas de Menadel e Daniel nota-se que essas sofrem por antecipação. Já nas falas de Vehuel e Aniel percebe-se o estresse e o medo quando vão a ESF para realizar o mesmo.

Apesar de a maioria das participantes estarem ciente sobre a importância do exame, de um modo sintético, elas ainda acabam procurando a UBS para realizar o mesmo, na maioria das vezes somente quando acometidas por alguma sintomatologia. Cerca de 74,97 \% das participantes afirmaram procurar a UBS para submeter-se o Papanicolau apenas quando apresenta algum sinal/sintoma. O MS preconiza que no caso do primeiro resultado do exame citopatológico apresentar "dentro dos limites de normalidade", deve-se repetir o mesmo em um ano. Após 2 resultados normais consecutivos o controle deverá ser feito a cada 3 anos, chamado esse de rotina de rastreamento. Algum resultado alterado e/ou sintomatologia/sinal deverá ser tratado seguindo o protocolo do MS (BRASIL, 2005)

75 Id en line Revista de Psicologia. Ano 9, No. 27. Julho/2015 - ISSN 1981-1179. Edição eletrônica em http://idonline.emnuvens.com.br/id 
Vale ressaltar que apenas 3 das 12 entrevistadas referiram que procuram realizar o exame anualmente, mesmo sem qualquer queixa:

“... estava com um corrimento amarelado...” MICAEL.

"Foi o agente de saúde que foi na minha casa e disse que a enfermeira do posto estava me chamando para fazer o exame...”. LEIAZEL.

“... já estou em uma idade avançada, vou entrar na menopausa, tenho que cuidar da saúde.” LECABEL.

"Os sintomas que eu estava sentindo irritação, muita coceira, mau cheiro, tipo manter relação sem tomar banho. ” MENADEL.

“Estou tendo corrimento branco sem cheiro...”. ANIEL.

“... procurei devido às dores que estava sentindo próximo a menstruar...” GABRIEL.

“... porque nunca tinha feito, desde os 16 anos tenho relação sexual e nunca tinha feito esse exame...” AREIL.

Diante dos achados percebemos uma busca ativa em relação à prevenção do exame de Papanicolau. Uma pesquisa feita por Fioravente (2008) sobre os fatores associados à realização dos exames preventivos de CA de mama e de colo uterino pelas mulheres brasileiras comunga-se com os resultados dessa pesquisa, quando o autor afirma que a maior associação com a realização do exame Papanicolau é quando as mulheres, em sua maioria, apresenta algum sinal/sintoma, em contrapartida o autor diz que diferente do exame, o exame das mamas é realizado como forma preventiva por essas mulheres, devida talvez a divulgação da mídia.

Já um estudo sobre o mesmo assunto, realizado por Braga et al (2006) revela em seus resultados, assim como o de Fioravente (2008), que a maioria das mulheres que realizaram o exame procuraram o serviço de saúde por motivo de doença e poucas são para atendimento relacionados a uma atitude preventiva. Um comparativo entre vários estudos revela que a realidade em comum das mulheres é que em suma, tratam o exame de Papanicolau como um exame diagnóstico e não preventivo.

O que chama a atenção é a falta, talvez, de incentivo para realização do exame de Papanicolau e a cultura de que o mesmo é um exame para detectar alguma alteração e não para prevenção.

\section{Considerações Finais}

Ao analisarmos o perfil socioeconômico das participantes, prevaleceram aquelas entre 18 a 28 anos de idade, $41,66 \%$, quanto ao estado civil prevaleceram às casadas, $91,63 \%$, a respeito do grau de 
escolaridade, a maioria se encontra com um nível baixe para sua idade (ensino fundamental incompleto). Quanto a profissão e renda familiar, prevaleceram as donas de casa e as que vivem com 1 salário mínimo mensal, respectivamente 41,66\% e 58,31\%. Comparando os dados socioeconômicos, notamos que há uma intima relação entre as condições econômicas não favoráveis e baixo grau escolar, formando assim um grupo suscetível.

Quanto ao perfil ginecológico predominou aquelas que diziam não se lembrar da quantidade de exames de Papanicolau já realizado, talvez pela demora de procurar atendimento na UBS, chamadas grupos de faltosas. Quando indagadas sobre os resultados dos exames de Papanicolau, referiram, a maioria, 83,03\%, que vem sempre com alguma alteração seja uma inflamação e/ou corrimento, segundo as participantes. Apenas 2 relataram que o resultado veio sem qualquer alteração. Neste estudo não foi detectado qualquer diagnostico de maligno ou metalepsia, de acordo com relatos.

Quando foram questionadas sobre a periodicidade do exame de Papanicolau foi expressivo o número daquelas que afirmaram só procurar a UBS quando apresentam algum sinal / sintoma, 49,88\% das entrevistadas. O que percebemos também é a forma aleatória com que realizam o exame, algumas realizam de 6 em 6 meses, outras anualmente, fugindo assim daquilo preconizado pelo MS. Ver-se a falta de informação sobre a periodicidade de realizar o exame. É necessário o esclarecimento e o incentivo em realizar o exame em questão.

Quanto à categoria conhecimento das mulheres acerca do exame de Papanicolau, prevaleceram aquelas que dizem saber que o exame é para diagnosticar doenças, referiam principalmente ao CA e as DST's. De uma forma resumida, elas demonstraram algum tipo de conhecimento sobre a funcionalidade do mesmo, mas não tem noção de sua periodicidade. Declararam também que tiveram informações dos vizinhos, amigos e/ou família sobre o assunto em questão. Não foi relatado, em qualquer momento da pesquisa, que foram passadas informações dos profissionais de saúde da unidade.

Ao analisarmos esses dados percebemos a não divulgação do objetivo real do exame de Papanicolau de acordo com o MS. Seria de extrema importância à divulgação pela mídia, órgãos responsáveis pelo programa e profissionais de saúde.

As entrevistadas foram indagadas sobre os fatores que influenciam a não adesão do exame, prevalecendo à timidez a justificativa de não realizá-lo. Outros dois fatores relatados foi o temor ao resultado e o incomodo ao realizar o exame. É sabido que esses fatores impeditivos são supridos com o diálogo e esclarecimento de dúvidas, antes de iniciar o procedimento. Sabe-se também que a confiança que a paciente tem no profissional faz com que ela sinta-se mais a vontade e relaxada, consequentemente o procedimento é mais ágil e menos incomodo para ela, deixando de lado sua timidez e/ou cultura. Vale ressaltar que não foi verbalizado pelas participantes que a não aderência ao mesmo e por falta de recursos materiais/humanos. 
Já na categoria de o que leva as mulheres a realizarem o exame de Papanicolau, foi unânime aquelas que afirmaram procurar a UBS apenas quando apresenta algum sinal/sintoma. Comungando-se talvez com os resultados prevalentes da não adesão ao exame, que é justificada por elas "a vergonha". Chama atenção à ausência da busca ativa daquelas faltosas, por parte da UBS e uma divulgação por parte do MS, assim como é bem divulgado o exame preventivo de CA de mama.

O câncer de colo uterino deve ser evitado, ou simplesmente imediatamente tratado, pelo simples fato de que as mulheres têm acesso fácil ao exame de Papanicolau, não tem altos custos para o governo e se detectado precocemente, tem cerca de $100 \%$ de chance de ser tratado com sucesso. O que falta apenas é um incentivo na divulgação do mesmo e conscientização da população, se há esta formação, essa consciência da realização do exame, os dados referentes ao grande número de casos encontrados em estágio avançados serão suprimidos.

Para tanto, é preciso trabalhar três pontos no processo de gestão do programa: 1) Autoformação continuada dos profissionais no aspecto de abordar a cliente; 2) Conscientização da comunidade quanto à importância do exame e sua forma de realização, assim como quem são os profissionais que realizam o mesmo exame; e 3) Promoção de campanhas educativas e de realização do exame em tempos específicos do ano, para chamar atenção da comunidade e ser, para o município, um período eficiente para realização de uma busca ativa de doenças na sexualidade feminina. Com estes, espera-se sim, ter uma melhor resolutividade da realização do exame, e uma aceitação considerável da comunidade em fazê-lo.

\section{Referências}

ALMEIDA, M. T. G.; et al. Câncer cervical: etiologia, diagnóstico e prevenção. Arq. Ciênc. Saúde, out.-dez.; 15(4): 199-204.

BRASIL. Conselho Nacional de Saúde. Resolução no 196/96. Decreto 93933 de janeiro de 1987. Estabelece critérios sobre pesquisa envolvendo humanos. Bioética, v. 4, nº 2, p. 15-25, [Suplem.], 1996.

BRASIL, Ministério da Saúde. Secretaria de atenção à saúde. Departamento de ações programáticas estratégicas. Área técnica de saúde da mulher. Pré-natal e puerpério: Atenção qualificada e humanizada -Manual técnico - Brasília: Ministério da Saúde, 2005.

Secretaria de Atenção à Saúde. Departamento de Atenção Básica. Controle dos cânceres do colo do útero e da mama. Brasília: Ministério da Saúde, 2006.

Ministério da Saúde. Condiloma Acuminado ou HPV. Disponível em: http://www.aids.gov.br. Acesso em: 12 de Nov. de 2010.

CAMPOS, A.C.C.; FREITAS-JÚNIOR, R.; POLETTO, K.Q.; GOULART, E.F.; RIBEIRO, L.F.J.; PAULINELLI, R.R.; REIS, C. Fatores de risco associados às alterações celulares induzidas pelo 
papilomavírus humano no colo uterino. In.: Rev. Ciênc. Méd., vol. 17 (3-6): 133-140. Campinas, maio/dez. 2008.

CARDOSO, E.J.F.; LIPPAUS, R. A enfermagem na prevenção do câncer do colo de útero. Curitiba. Disponível em: http://www.scielo.org. Acessado em: 19 de set. de 2010.

CERVO, A.L.; BERVIAN, P.A. Metodologia científica. 5. ed. São Paulo: Prentice Hall, 2002.

CRUZ, L.M.B.; LOUREIRO, R.P. A comunicação na abordagem preventiva do câncer do colo do útero: importância das influências histórico-culturais e da sexualidade feminina na adesão às campanhas. In.: Saúde Soc., vol. 17 (2): 120-131. São Paulo: 2008.

DAVIM, R.M.B.; TORRES, G.V.; SILVA, R.A.R.; SILVA, D.A.R. Conhecimento de mulheres de uma unidade básica de saúde da cidade de Natal/RN sobre o exame de Papanicolau. In.: Re. Esc. Enferm. USP, vol. 39 (3): 296-302, 2005.

DUAVY, L.M.; BATISTA, F.L.R.; JORGE, M.S.B.; SANTOS, J.B.F. A percepção da mulher sobre o exame preventivo do câncer cérvico-uterino: estudo de caso. In.: Ciência e Saúde Coletiva, vol. 12 (3): 733-742, 2007.

DUTILH, H.M.N.; BRAGA, P.E.; SCHOUT, D. Fatores associados à realização de exames preventivos para câncer nas mulheres brasileiras, PNAD 2003. In.: Ciência e Saúde Coletiva, vol. 11 (4): 1023-1035. Rio de Janeiro: out./dez. 2006.

FERNANDES, J.V.; et al. Conhecimentos, atitudes e prática do exame de Papanicolaou por mulheres, Nordeste do Brasil. Rev. Saúde Pública, vol. 43 (5): 851-858, 2009.

FERREIRA, M.L.S.M. Motivos que influenciam a não-realização do exame de Papanicolaou segundo a percepção de mulheres. In.: In.: Esc Anna Nery Rev. Enferm., vol. 13 (2): 378-384, abr./jun. 2009.

GERK, M.A.S. Prática de enfermagem na assistência ginecológica. In.: BARROS, S.M.; MARIN, H.F.; ABRÃO, A.C.F.V. Enfermagem, obstetrícia e ginecologia: guia para a prática assistencial. São Paulo: Roca Ltda., 2002.

GREENWOOD, S.A.; et al. Motivos que levam mulheres a não retornarem para receber o resultado do exame Papanicolau. Rev. Latino-am. Enfermagem, vol. 14 (4): 503-509, jul./ago. 2006.

HANDEM P.C, et al Metodologia:Interpretando os autores In: FIGUEREDO,NÉBIA. M.A de: Método e Metodologia na pesquisa científica. $3^{\circ}$ ed.São Caetano do Sul,São Paulo:Fendes editora,2008.

IMEBI (Instituto de Medicina Biomolecular). Exame anatomopatológico: Papanicolau. Disponível em: 〈http://www.imebi.com.br/exame_anatomopatologico. php〉. Acesso em: 23 maio 2010.

INCA (Instituto Nacional do Câncer). Câncer do colo do útero. Comunicação Social, 2009. Disponível em: 〈www.inca.gov.br $>$. Acesso em: 23 maio 2009.

LEOPARDI, M.T. Metodologia da pesquisa na saúde. Santa Maria: Pallotti, 2002.

LIMA, C.A.; PALMEIRA, J.A.V.; CIPOLOTTI, R. Fatores associados ao câncer do colo uterino em Propriá, Sergipe, Brasil. In.: Cad. Saúde Pública, vol. 22 (10): 2151-2156. Rio de Janeiro: out. 2006. 
MARCONI, M. A; LAKATOS, E. M: Fundamentos de metodologia científica. 6 aed. São Paulo:Atlas, 2005.

MARCONI, M. A; LAKATOS, E. M. Metodologia científica. 5aed. São Paulo: Atlas, 2009.

MINAYO, M.C.S.O. O desafio do conhecimento: pesquisa qualitativa em saúde. 11. ed. São Paulo: HUCETEC-ABRASCO, 2007.

MONTENEGRO, C.A.B.; REZENDE FILHO, J. Obstetrícia fundamental. 11. ed. Rio de Janeiro: Guanabara Koogan, 2008

MORAES, M.W. Câncer ginecológico. In.: MOLHALlEM, A.G.C.; RODRIGUES, A.B. Enfermagem oncológica. São Paulo: Manole Ltda., 2007.

NADAL, S. R.; MAZIONE, C. R. Vacinas contra o Papilomavírus Humano. Rev. Bras. Coloproct. v. 26, n 3, p. 337-340, Jul.-Set., 2006.

OLIVEIRA, M. M de. Como fazer pesquisa qualitativa. $2^{\text {a }}$ Ed.Rio de Janeiro:Vozes, 2008.

OLIVEIRA, M.M.H.N; et al. Cobertura e fatores associados à não realização do exame preventivo de Papanicolaou em São Luís, Maranhão. In.: Rev. Bras. Epidemiol., vol. 9 (3): 325-334, 2006.

PIATO, D.S.A.M.; PIATO, J.R.M.; CARVALHO, J.P.; SOUEN, J.S. Câncer de colo do útero. In. PINOTTI, J.A.; FONSECA, A.M.; BAGNOLI, V.R. Tratado de ginecologia: condutas e rotinas da disciplina de ginecologia da Faculdade de Nedicina da Universidade de São Paulo (USP). 2. ed. São Paulo: Revinter Ltda., 2005.

RAMOS, A. S.; et al. Perfil de mulheres de 40 a 49 anos cadastradas em um núcleo de saúde da família, quanto à realização do exame preventivo de Papanicolaou. In.: Rev. Latino-am. Enfermagem, vol. 14 (2): 170-174, mar./abr. 2006.

RIVOIRE, W.A.; REIS, R.; MONEGO, H.I.; APPEL, M.; CAPP, E. Carcinoma invasor de colo uterino. In. FREITAS, F.; MENKE, C.H.; RIVOIRE, W.A.; PASSOS, E.P. Rotinas em ginecologia. 5. Ed. Porto Alegre: Artmed, 2006.

RIVOIRE, W.A; MONEGO, H.I.; MAFNO, V.; APPEL, M.; REIS, R.; CAPP, E. Câncer e gestação. In.: FREITAS, F.; COSTA, S.H.M.; RAMOS, J.G.L.; MAGALHÃES, J.A. Rotinas em obstetrícia. 5. ed.Porto Alegre: Artmed, 2006.

ROBBINS; et al. Patologia estrutural e funcional. 6 ed. Rio de Janeiro: Guanabara Koogan, 2000.

SANTANA, E.A.; BISELLI, P.M.; BISELLI, J.M.; ALMEIDA, M.T.G.; BERTELLI, E.C.P. Câncer cervical: etiologia, diagnóstico e prevenção. In.: In.: Arq Ciênc Saúde, vol. 15 (4): 199-204, out./dez. 2008.

SANTOS, J.O.; SILVA, S.R.; SANTOS, C.F.; ARAÚJO, M.C.S.; BUENO, S.D. Alterações cérvicouterinas em mulheres atendidas em uma unidade básica de saúde no município de Campinas-SP. In.: REME - Rev. Min. Enf., vol. 11 (4): 439-445, out./dez. 2007.

SANTOS, M.L.; MORENO, M.S.; PEREIRA, V.M. Exame de Papanicolaou: qualidade do esfregaço. In.: Revista Brasileira de Cancerologia, vol. 55 (1): 19-25, 2009. 
SILVEIRA, L.M.S.; VERAS, R.C.; CRUZ, A.L.N.; FARIA, M.S. Gestação e papilomavírus humano: influência da idade materna, período gestacional, número de gestações e achados microbiológicos. RBAC, vol. 40 (1): 43-47, 2008.

SOARES, M.C.; et al. Câncer de colo uterino: caracterização das mulheres em um município do sul do Brasil. Esc Anna Nery Rev. Enferm., vol. 14 (1): 90-96, jan./mar. 2010.

TERMINI, L.; VILLA, L.L. Biomarcadores na triagem do câncer do colo uterino. In.: DST - J. Bras. Doenças Sex. Transm., vol. 20 (2): 125-131, 2008.

YASSOYAMA, M.C.B.M.; SALOMÃO, M..L.M.; VICENTINI, M.E. Características das mulheres que realizam exame preventivo do colo do útero durante a gestação: bases para estratégias do Programa de Saúde da Família (PSF). In.: Arq Ciênc Saúde, vol. 12 (4): 172-176, out./dez. 2005.

\section{Como citar este artigo (Formato ABNT):}

ALMEIDA, S.L.; MATOS, S.K.C.; PAZ, C.B.; SANTANA, O.M.L.L.; CARVALHO, C.M.L.; BARBOSA, J.S. Fatores Relacionados à Adesão do exame de papanicolau entre as Mulheres de 18 a 59 anos de Idade. Id on Line Revista de Psicologia, Julho de 2015, vol.9, n.27, p. 64-81. ISSN 1981-1189.

Recebido: 04/05/2015

Aceito: $12 / 05 / 2015$ 